\title{
Some Direct and Indirect Interactions of Weather and Topography with Resistance to Disease in Animals
}

\author{
by \\ N.St.G. Hyslop
}

\section{INTRODUCTION}

Our understanding of the complicated relationships between the multiple biosystems which are involved in disease processes have changed remarkably during recent years. It has become evident that in many conditions, of both infectious and non-infectious etiology, a large number of endogenous components and exogenous influences, involving both the environment and the "host", interact through complex synergistic or antagonistic interrelationships to produce results ranging between clinical disease, subclinical disease or the continuance of the healthy state.

In evaluating "immunity" we can no longer confine our attention solely to the classic humoral immunity, manifested by circulating antibodies, nor even to the combined effects of humoral and cell-mediated immunity (CMI) but, rather, we must consider all the mechanisms which constitute barriers to the entry of foreign substances and to the replication of infectious agents.

Species, racial, other genetic, anatomic, physiologic, humoral and cellular factors establish the basic immune status of individual human beings and of animals. The variability of resistance demonstrable in species, strains and individuals within a taxonomic group of animals may depend partly on the possession of adequate nonspecific defence mechanisms, including the ability to produce substances such as lysozymes, lysins, properdin and other microbial inhibitors, transfer factor, and other activators of host defence mechanisms. The basal immune status is influenced further by past and present exposure to parasites, to toxins, and to pathogenic organisms distributed throughout the biosphere; it is modified also by climatic, dietary and stress factors. The environment has been characterized as consisting of mainly favourable (nurturing) or unfavourable (stressor) influences: the response of all Living organisms to these influences depends partly on genetic factors and partly on their interaction with "positive" or "negative" ecological components.

With the exception of certain developing countries, in which disruption of disease control measures caused by wars and political unrest has resulted indirectly in some increase in the major epizootic diseases, a gradual change has been noted nearly everywhere in the general patterns of animal disease during the past 30 years. It appears that wherever "sanitary-police" measures are now adequate, the more acute manifestations of sickness are being supplanted by complex and insidious forms, often associated with relatively low mortality but high morbidity and a consequent serious loss of potential productivity. Much of this economic loss can be attributed, directly or indirectly, to exacerbation of "mild" infections or adverse metabolic functions by environmental stress. A particularly good example of the interaction of environment and disease processes of this type is seen in the group of respiratory diseases which occur in young cattle maintained under unnatural or sub-optimal conditions of husbandry. Nevertheless, it is uncertain whether respiratory or enteric diseases cause the greater financial loss. The increasing number of bacteria, mycoplasmata and viruses which have been isolated from animals manifesting clinically similar syndromes, together with the usually inconclusive results of attempts to fulfil Koch's postulates with any single organism, suggest that the pathogens probably act synergistically and that environmental factors play an important role in the outcome.

Some idea of the magnitude of the economic implications of all infectious and non-infectious diseases of livestock, in the USA alone, is indicated by the estimate that annual losses in the period 1951-1960 were at least $\$ 1,422,020,000$. More than 50 of these diseases are known to be affected by seasonal, climatic or environmental factors. Climate, weather and topography may influence the immune status of entire populations by direct effects of stress, by causing dietary deficiencies through their effects on crops etc., by spreading waterborne or airborne pathogens, by necessitating the movement of diseased livestock, and by causing extension or contraction of zones occupied by arthropod and other disease vectors. 


\section{PHYSICAL EFFECTS}

\section{A. STRESS}

It is well established that neural stimuli, often operating for long periods at little more than threshold levels, reach the hypothalamus and activate certain factors, which in turn stimulate the adenohypophysis of the anterior pituitary to cause release of adrenocorticotrophin (ACTH) into the general circulation. In the higher mammals, when this polypeptide hormone arrives at the adrenal gland, it stimulates synthesis of the steroid hormones cortisol and cortisone (corticosterone in lower mammals and birds). The latter are thought to bind to specific cytosol proteins in "target tissues", thus producing a change in the synthesis of messenger RNA. This modification mediates synthesis of different cell-proteins and evokes a functional response characteristic of the tissue involved. In many animals an additional direct neural stimulation of the adrenal medulla probably occurs, with subsequent release of the catecholamines adrenalin and noradrenalin. The release of catecholamines, in particular, appears to play an important role in the regulation of the immune response through inhibition of cyclic adenyl-monophosphate, whilst glucagon mobilizes glycogen stores. Complex interactions of androgens probably produce secondary effects.

Endocrine balance influences the rate of maturation of the cells of the immune systems as well as the immunological status during a period of stress. Somatotrophin, adrenal glucocortico-steroids, and their analogs, exert a profound influence on the number and function of circulating lymphocytes of both the $\mathrm{T}$ (thymus-dependent) and the $\mathrm{B}$ (bone marrow-derived) series, and may produce a marked lymphopenia in some animal species. However, a proportion of these cells (i.e. steroidresistant lymphocytes) may be driven into the bone marrow and other "refuge areas", whence they may emerge again later. The rate of antibody production and the level of antibody in circulation may not always be altered very rapidly by the presence of steroids, but CMI is usually inhibited promptly (within minutes) by direct depression of macrophage metabolism and by blocking of the shortrange soluble mediators of CMI (iymphokines) which are liberated by activated T-lymphocytes. It must be emphasized, however, that very recent observations reveal there are marked differences in the sensitivity of different mammalian Orders to the effects of steroids, and so response to the hormonal effects of environmental stress in one animal may differ from that of an animal of a different Order. Furthermore, when corticosteroids are released at relatively low levels, certain immune functions may be actually enhanced, rather than depressed, possibly because of elimination of mononuclear cells of types irrelevant to the immediate defence requirements of the animal. However, as a generalization, it may be stated that stimulation of the pituitary-adrenal system during stress results in a diminution of the defences provided by the "non-specific" inflammatory responses, depression of CMI and, eventually, depression of the humoral immunologic processes.

Another effect of high levels of corticosteroids on the immune processes of cattle was reported recently when Australian workers demonstrated that these hormones reduced the secretion of $\operatorname{IgG}_{1}$ into the colostrum of pre-parturient cows. It will be remembered that, whilst $\operatorname{Ig} A$ is the principal source of colostral antibody for human infants, the main sources for calves are the Ig Gs. Thus, calves may receive only a reduced degree of protection when corticosteroid levels in the dam are high. Furthermore, it has been demonstrated by several investigators that the susceptibility of colostrum-deprived calves to calf diarrhea, a disease of complex aetiology, is greatest in the period from late fall to early spring. Likewise, high mortality has been recorded frequently in the lambs of ewes which have been exposed to harsh weather shortly before parturition.

When extremes of climate or rapid change in weather patterns cause severe stress, and especially when topographic factors impose a requirement to travel long distances over rough terrain in the search for food and water, the effects of stresses of different types tend to be cumulative. Somewhat similar stress mechanisms probably operate under conditions of intensive husbandry in overcrowded premises and damp insanitary conditions, and these effects may be exacerbated by psychologic pressures.

Stress and fatigue increase significantly the persistence of bacteria in the alimentary tract. These factors have been shown to increase morbidity and mortality from poliovirus in experimental animals, and have been incriminated as adverse factors in the epidemiology of human poliomyelitis. Similarly, epizootiological studies on contagious bovine pleuropneumonia provide a classic example of the effects of general stress in the field. Clinically normal cattle, originating in endemic areas of Africa or Australia and carrying inapparent foci of infection, often developed acute pleuropneumonia during transit to market, sometimes spreading the disease into areas from which it had already 
been eradicated. The resurgence of infection is nearly always associated with herding across long distances under adverse climatic conditions, especially high temperature and drought. Similarly, heat, cold, trauma, emotional disturbances or excessive exercise, especially in asthmatics, may impair pharmacological and immune mechanisms in man, and certain patients may develop immune responses, possibly as a result of the release of "altered-tissue" antigens to which the patient has become sensitized. Undoubtedly, adrenal dysfunction plays an important role but it is becoming evident that seasonal variations combine with dietary and hormonal influences to link stress with immune processes by extremely complex mechanisms.

Heat is not the only cause of stress under tropical conditions. Furthermore, tropical conditions tend to favour the spread of certain diseases, and of disease vectors. For these reasons, wide variations in immune status may be found in the populations of contiguous localities in tropical zones.

\section{B. TEMPERATURE AND HUMIDITY}

High temperature and low relative humidity $(\mathrm{RH})$, as well as irritants such as $\mathrm{SO}_{2}$ and other air pollutants, all exert a direct effect on the "non-specific" defenses of the respiratory and gastrointestinal tracts. Sudden changes in environmental temperature may have especially profound effects. Because of condensation and precipitation, prolonged periods of very low temperature are associated with low RH; the latter factor has been shown to exert further unfavourable effects on the lining cells of the respiratory tract and may impair ciliary clearance mechanisms. Intense chilling modifies the mucous secretions and may cause submucous oedema.

In addition to a direct influence on the ability of infective agents to proliferate in the body, high temperatures increase the metabolic rate of the host. However, there is some evidence that high temperature does not always accelerate humoral responses to non-replicating antigens or to some bacteria, and high fever not only impairs active immunization but accelerates the regression of existing antibody titres. To cite but two examples, in comparison with controls maintained at moderate temperature, mice maintained at $6-10^{\circ} \mathrm{C}$ were found to be more resistant to tetanus toxin, and those kept at $35^{\circ} \mathrm{C}$ were less so, though animals at the lowest temperature were capable of making the least humoral response to inactivated toxoid. Similarly, swine maintained at a constant temperature of $30^{\circ} \mathrm{C}$ and infected experimentally with Erypsipelothrix developed greater temperatures and more severe clinical reactions than control animals kept at $13-15^{\circ} \mathrm{C}$. Those maintained at the higher temperature showed a decrease in blood ascorbic acid levels, in serum complement, in phagocytic index and in circulating eosinophils. Cold has been shown also to reduce the activity of macrophages against numerous organisms including Pasteurella hemolytica . It is probable that, in numerous diseases, this decreased macrophage-processing at subnormal temperatures may have a profound effect on the total immune response. The generally adverse effects of cold have been observed also in relation to the severity and the course of trypanosomal infections.

The situation relating to viral infections appears to be quite different, and depends partly on the nature of the virus. Maintenance at about $36^{\circ} \mathrm{C}$ tends to prolong the survival of animais infected with the viruses of poliomyelitis and myxomatosis, with Coxsackievirus type B and with other viruses. Coxsackie virus $\mathrm{B}$, though almost invariably lethal for mice maintained at $4^{\circ} \mathrm{C}$, usually causes severe disease in those at $25^{\circ} \mathrm{C}$, but only inapparent infection in mice kept at $36^{\circ} \mathrm{C}$. However, both increase and decrease of temperature, as well as stress or intercurrent disease, are well recognized as important factors in reactivating latent infection with herpes simplex virus in both man and animals. Moderately low temperatures, especially in association with a lack of light and high $\mathrm{RH}$ in confined living accomodation, exert well-known adverse effects on the health of man. Similar effects are noted frequently among young animals, such as calves and pigs, which also may be deprived of colostrum and may be maintained in insanitary and overcrowded conditions. The vital importance of indoor climate in pig breeding establishments has been demonstrated by many authors thus there may be a tendency towards an increased incidence of weak piglets and mortality from respiratory diseases during the winter months, but an increase of enteritis in the summer and autumn. Air velocities as low as 0.2 metres/sec may chill piglets, though older animals may be able to tolerate draughts of somewhat greater velocity without impairment of general health.

The influence of intense cold on disease patterns in isolated communities has been well illustrated by clinical and serological studies, with special reference to corona, influenza and Coxsackie viruses, among personnel living for long periods in the Antarctic bases. In general, resistance appeared to wane during several months of isolation but, nevertheless, parties remained free from colds except when visited by stores ships: men who boarded ships to return home often developed colds 
soon after initial contact with outsiders. Occasionally, however, symptoms developed in isolated parties after a severe fall in outside temperature. Of course, this suggests that a latent infection may have been reactivated and then overcame the declining residual immune mechanisms.

In retrospective studies, seasonal fluctuations in ESR, albumin and gamma-globulin levels, statistically correlated with the "cooling-index" of the atmosphere, have been associated with changes in resistance to infection. Thus, a reduction in gamma-globulins has been advanced as one explanation for an increase of respiratory disease among persons who have recently returned to Europe after holidays in warm climates. It is sometimes difficult to explain why, when weather conditions appear to favour the development of epidemics, small outbreaks often merely die away. The importance of coincidence in time of pathogen and weather has been stressed and it is postulated that "if a suitable agent is present in a community, a serious epidemic situation is possible when two periods of inclement weather are separated by a time interval equal to the incubation period of the disease".

The relationship between seasonal climatic variations and immunity in hibernating animals constitutes a special case. During hibernation, both cellular activity and antibody production are partly inhibited but, nevertheless, resistance to bacterial, protozoan and metazoan parasites tends to increase. Although these observations have been confirmed frequently it is possible, having regard to reduced antibody production, that increase in apparent resistance partly reflects a greater sensitivity to cold by the parasite than by the hibernating host. When infection remains latent, for example with rabies virus or Coxsackie viruses in the brown fat bodies of certain animals, clinical disease may develop on re-awakening; alternatively, as in the case of leptospiral infections, some animals may develop an immunity sufficient to prevent reinfection, but remain symptomless carriers for long periods.

Vaccination may be impaired by high or low ambient temperatures. Thus, it is evident that one must distinguish between the effects of temperature on resistance and on immunizability. Since climate exerts extremely complex influences on humoral and cellular immunity, this remains a field for much intensive research.

\section{ALTITUDE AND OXYGEN-TENSION}

The remarkable responses to low $\mathrm{O}_{2}$ tension possessed by most mammals, including man, tend to reduce the effects of high altitude after a period of acclimatization. During short periods of anoxia, there is some general propensity for the phagocytic mechanisms to be inhibited, whilst antibody production may be enhanced, at least temporarily. The effects of hypoxic stress cannot be excluded as a contributory factor, but it is probable that, under conditions of prolonged exposure to low concentrations of oxygen, the erythropoietic and lymphopoietic tissues compete for pluripotential stem cells, with the result that precedence is given to the need to increase the number of oxygentransporting erythrocytes in circulation. Consequently, differentiation of stem cells into immunocytes is wholly or partly blocked, and resistance to infection may decline until oxygen requirements are satisfied. Very large differences in circulatory immunoglobulin levels have been reported in closely related ethnic groups living at different altitudes in equatorial zones.

\section{NUTRITIONAL EFFECTS}

The environment influences nutritional levels of man and animals, not only (a) by means of organic and inorganic soil constituents in the particular area, and (b) by the availability of prey species for carnivores, but also (c) by seasonal and longer-term fluctuations in temperature, rainfall, etc. The extent to which environment-mediated nutritional factors, in turn, influence immune processes remains to be determined fully. However, a close association between famine and pestilence has been remarked for many centuries. An ever-increasing volume of experimental and observational evidence suggests that humoral immunity at least, and probably cellular immunity also, depend to a great extent on maintenance of the nutritional plane within fairly narrow limits.

In bacterial diseases, aithough diet may not always affect the frequency of infection, the ability of patients to resist the most severe effects of disease is notably enhanced when dietary levels are good or adequate, whereas even short periods of starvation have been shown to impair resistance to invasion by organisms such as Mycobacterium tuberculosis and Salmonella of various species.

Numerous (but not all) studies reveal a loss of resistance with progressive protein depletion, and avitaminosis has been linked experimentally with low resistance to infection. Vitamins $\mathrm{A}, \mathrm{C}$ and $\mathrm{E}$ appear to be particularly important, but it is beyond the scope of the present review to discuss 
recent incompletely substantiated claims for prophylaxis or therapy by massive doses of specific vitamins. Scrimshaw and his co-workers have listed in detail instances of the interaction of dietary deficiencies in bacterial diseases, and the interested reader might profitably consult this source.

By contrast, in diseases of viral aetiology, the effects of dietary deficiency are variable and often contrary to those encountered in bacterial infections. Perhaps this disease-retarding effect is attributable more to poor "cellular tone", and to a lack of particular intracellular metabolites on the replication of causal agents, than to the direct action of diet on humoral immunity mechanisms . Thus, we have encountered relatively severe reactions to imcompletely-attenuated rinderpest vaccines (KAG), among unvaccinated cattle maintained on a high plane of nutrition; whereas the same batch of vaccine evoked only mild or undetectable reactions among herds of the same breedtype, which were grazing very poor herbage in drought-stricken areas of Africa where rinderpest had not occurred for many years. However, it must be remembered that intercurrent infestation of animals with ticks and protozoa probably plays an important part in reactions to rinderpest virus, and such infestations are themselves mediated by climatic and topographical factors. Similarly, I found, during experimental exposure of non-immune steers to aerosols of the herpesvirus of infectious bovine rhinotracheitis (IBR), that a greater proportion of severe clinical cases developed among animals in good general conditions that among animals which had been maintained on a poor diet. In the field, however, a somewhat different situation generaily prevails, with the result that concurrent infection of undernourished calves with IBR virus and adventitious pathogenic bacteria usually results in synergism of the pathogens and a consequent severe morbidity. Enhancement of CMI by chronic protein deficiency will be discussed later.

Environmental and nutritional influences appear to play an important but incompletely determined role in the pathogenesis of chlamydial and rickettsial diseases, such as typhus in man and heartwater fever in animals.

The effects of multiple dietary deficiencies, often combined with exposure to cold and damp, facilitate the infestation of lambs and calves with quite a wide range of gastro-intestinal nematodes such as Trichostrongylus, Ostertagia, Cooperia, Nematodirus and lungworms such as Dictyocaulus spp

Thus nutritional deprivation also exacerbates hookworm infestation of man, and probably, of animals; similar effects have been noted with Ascaris, Trichinella spiralis and a number of other parasites.

The situation regarding protozoa is less clear. The deficiency of Vitamin A has been shown to increase the susceptibility of rats to Trypanosoma cnizi and, in general, dietary deficiencies exacebate amebiasis and other intestinal infestations. But high protein intake may tend to inhibit the effects of blood-borne parasites, such as Plasmodium falciparum, P.berghei, and Trypanosana congolense in animals. As an example T.congolense normally requires up to one month to produce

marked clinical effects in healthy cattle on good pastures; however, the incubation period is reduced to as little as 10 days in cattle living under exceptionally harsh conditions on poor grazing. Furthermore, the clinical course of this and many other trypanosomal infections is greatly influenced by adverse environmental factors such as heat and drought. In the latter examples we are probably considering the combined effects of dietary and physical stress, both of which resulted from environmental factors related to topographical features.

The precise relationship between diet, resistance to infection and the production of humoral antibody requires very intensive investigation, and is currently the subject of study by a Consultation Group sponsored by the World Health Organization. Early studies showed that dogs whose serum proteins had been depleted experimentally were very susceptible to disease, but regained considerable resistance when given a high-protein diet. Part of the problem lies in the fact that deficiencies seldom occur as single entities. In particular, adequate supplies of protein, fat, vitamins and, possibly, trace elements appear to be essential for rapid response by the immune mechanisms of all mammals. Moreover, a properly balanced diet may be as important as the absolute values of constituents, provided of course that the basal adequacy threshold has been attained. Below this threshold, leukocyte maturation may be impaired, as in kwashiokor, and a profound lymphopenia may be detectable in severely malnourished animals; thus, mice kept on a low protein diet make fewer antibody-forming cells than mice on a balanced diet. Recent studies on human subjects in South America and in South Africa have revealed a similar picture of lymph node and thymic hypoplasia; lymphopenia may be evident in some cases. However, there is evidence that the moderately malnourished individual may still be able to direct protein synthesis preferentially towards immunoglobulin production at least for some time and, especially, to the production of Ig G (though possibly not Ig A and Ig M). Not all of this preferentially-synthesized protein can be demonstrated to possess a true antibody function. The complexity of the relationship between diet 
and immunologic response, and the necessity for a balanced diet, has been exemplified further by recent observations on New Zealand black mice, which tend spontaneously to become Coombspositive and to develop antinuclear factors. In these animals, the proportion of fat in the diet modified immune processes to an marked degree.

The mechanisms by which the influence of the environment on diet affects immunity remain to be determined fully, but it now seems clear that, in adults of most species, many nutritional deficiencies depress reticulo-endothelial activity as a whole, thus affecting both phagocytic action and antibody production. Malnutrition leads first to a fall in total serum proteins (especially albumin) and eventually to a severe hypo-gammaglobulinaemia; but again it must be emphasized that gammaglobulin levels alone do not necessarily reflect resistance to infection. It is of particular interest to note that a relationship has been found between Vitamin $\mathrm{C}$ and a thymic humoral factor (thymosin) which mediates the maturation, growth, regeneration and immunologic function of lymphoid tissues. It is significant also that Vitamin A in moderate doses (administered for several days at about the time of contact with antigens) reveals a marked adjuvant effect in mice, so that the humoral response is increased and CMI is stimulated. These effects are associated with depletion of lysosomes and a consequent increase in the cellularity of regional lymph nodes; however, acute hypervitaminosis A may exert a depressant effect on some forms of immunologic response.

The effect of dietary deficiency under extreme conditions has been investigated in germ-free animals (principally rats and mice) raised on sterile chemically-defined diets of low molecular weight, and similar effects are seen occasionally in human beings. Under conditions of starvation, there is some degree of thymic atrophy and cell depletion occurring in the thymus-dependent paracortical areas of the lymph nodes, so that lymphoid tissues are diminished in size and apparent activity. In the extreme state, there is slow or undetectable production of gammaglobulins. Nevertheless, despite lack of stimulation by dietary antigens, which might be expected to exert a trophic effect on total antibody production, such animals are generally capable of making some response to strong antigenic stimuli unless, of course, they become rapidly overwhelmed by an infective agent.

It has been shown by several workers that greater susceptibility to infection, consequent upon prolonged protein-calorie malnutrition, can be correlated with a decline in delayed hypersensitivity responses, and that the increased susceptibility is associated with an inability to synthesize the dialysable low molecular-weight transfer factor. This factor is derived from the leukocytes, and acts as an informational agent to regulate the status of CMI. However, the precise role of transfer factor in the response of the individual to infection remains to be elucidated fully, because it possibly possesses both activating and suppressive effects in different circumstances. Further influences of malnutrition have been attributed to depression of lymphocyte function by an increase in C-reactive protein and by a progressive fall in serum transferrin.

It is noteworthy that, in addition to their effects on the primary immune response, nutritional factors may affect the ability of the subject to develop a potent anamnestic humoral reaction, even after repeated exposure to an antigen. For example, not only may the primary response of rats to diphtheria toxoid be impaired if their diet is deficient in pyridoxin, pantothenic acid, biotin, or certain amino acids, but their anamnestic responses to the same antigen may be impaired still more markedly, even though their diet has not been deficient during the primary phase of sensitization.

In normal animals, the production of humoral (blocking) antibody tends to inhibit CMI, whereas, at least in the earlier stages, moderate to severe dietary deficiency (especially chronic protein deprivation) may often enhance cell-mediated immunity, partly by shutting off the production of blocking antibody, and partly by either increasing the responsiveness of the T-lymphocytes of by modifying the activity of "helper" macrophages.

It has been noted that peritoneal macrophages of mice on a protein-deficient diet ingest significantly more Listeria monocytogenes cells than do those of normal mice. However, reduction of protein below 3 per cent of the total calories depresses both humoral and cellular immunity, and death usually follows. Much earlier work also suggested that severe malnutrition decreases the antrgen processing action of macrophages upon bacteria, so that both CMI and humoral immunity are depressed. It can only be postulated that a "malnutritional threshold" occurs and, clearly, the interaction of various factors on macrophage performance requires further study.

It is evident that malnutrition beyond a certain point has similarly adverse effects on the cells of ciliated epithelium, on lysozyme production, and on the various other "non-specific" defence mechanisms in general. 
Prepartum malnutrition of the mother may have profound effects on the serum protein profile of the offspring. In dogs, these effects may persist almost to adulthood, and somehwat similar inability to resist infections later in life have been reported in rats. Incomplete immuno-competence may be associated with hypoplasia of the thymo-lymphic system, and several investigators have suggested that malnutrition of the dam during pregnancy, or malnutrition in the immediate postpartum period, may cause an arrest of the thymus-dependent lymphocyte system amounting to "nutritional thymectomy" in the progeny. Some interactions between the genetic constitution of breeds of cattle, environmental factors and diet were illustrated by experiments involving the exchange of calves in subtropical areas of South Africa, although the special parameter of diseaseresistance was not investigated. The weaning weights of Hereford calves were found to be low in comparison with those of the progeny of crossbred indigenous cows; and the calves of indigenous Bonsmara cows performed poorly when they were fostered by Herefords. However, when Hereford calves were suckled by the cross-bred cows, satisfactory preweaning weights were recorded. The difference in weaning weights was attributed to the inability of the Hereford dams to yield adequate amounts of milk under the prevailing climatic conditions.

Until now we have considered the effects of malnutrition and I have not mentioned the effects of overfeeding. Relatively little work has been reported on the immunologic effects of an excess of food, or of specific dietary components, though it is generally found that mammals which are overfed tend to possess a reduced resistance to infection. Thus, dogs on a high calorie diet are more susceptible to canine distemper, to viral hepatitis and to Salmomella spp. . than control animals on a normal diet. Similarly, in pigs a change to a protein-rich diet has been associated with the development of a moderate increase in gamma-globulins and the appearance of signs of arthritis and glomerulonephritis. It has been suggested that these changes result from an increase in the number, and an alteration in the nature of the organisms in the intestinal flora. In particular, certain strains of Clostridium perfringens flourish rapidly, and the subsequent passage of antigenantibody complexes into the blood appears to evoke a condition somewhat similar to rheumatoid disease in man.

Whilst the foregoing observations appear to be generally true for all species, nutritional requirements differ between various species so that semi-quantitative results can be applied only to the species in which the experiments were made. Furthermore, it must be remembered that genetic and familial variation may play a determinant role in the ability of a recipient's defences to respond fully to any particular antigen.

\section{EFFECTS OF ENVIRONMENTAL CONTAMINATION}

\section{A. NATURAL ANTIBODIES ETC.}

Possession of humoral resistance to a wide range of pathogens, by adults who have never exhibited corresponding clinical diseases, has been recognized for more than 80 years. These "natural" antibodies often appear to be identical in biochemical and biological characteristics with antibody evoked by clinical disease or by immunization. Absorption tests on such antibodies to enteric organisms have demonstrated a high degree of specificity. In the young, part of these antibodies may be of maternal origin, but in adults their incidence generally reflects the prevalence of the homologous organism in the environment. Surveys for Salmonella in the alimentary tract and in regional lymph nodes of normal animals have revealed an incidence of $2-10 \%$. Given such a high incidence and the probability of excretion, to say nothing of the possibility of additional low-level stimulus by dead organisms and by other agents possessing common antigenic determinants, it is scarcely surprising that the presence of "natural" antibody (of both 7S and 19S types) has been widely attributed to simuli of environmental origin. However, it must be remembered that the detection of such antibody does not always provide evidence of good immunity to reinfection. In man, a gradual increase in antibody titres has been demonstrated against a wide range of pathogenic organisms, including Pseudomonas aeruginosa, from childhood to adult life, whilst the presence of other antibodies is attributable to relatively nonpathogenic organisms and to dietary sources of antigen. The gamma globulins usually reach a peak during the $3 \mathrm{rd}$ decade and then decline slowly. Although breed variations occur, serum protein and serum gammaglobulin in dairy cows also have been shown to increase with age, but not all of the increment represents specific antibody.

\section{B. SPECIFIC ACTIVE INFECTION AND SENSITIZATION}

The degree and duration of contamination of the environment by pathogenic organisms is modified by climatic factors, whilst local geographic circumstances contribute to the frequency with which 
surviving organisms are brought into contact with susceptible populations. In all species, the continuing burden of infection throughout life plays an important part in the maintenance of individual and herd immunity. However, it is often difficult to relate the results of epidemiological studies to the constantly changing environmental data. Some indication of possible contamination rates is provided by the calculated inflow of 50 million Salmonella organisms per second into the river Rhine, and other rivers are probably equally contaminated. Contamination of food and water by enteric organisms from sewage or fecal slurries has been incriminated on innumerable occasions in the development of resistance among local populations, and the role of airborne organisms in acute disease has received an increasing amount of attention in recent years.

Nevertheless, one of the greatest and most intractable problems of immunology is presented by the vast numbers of pollens, spores and other vegetable fragments circulating in the ambient air. Their involvement in the induction of chronic allergic states, which may be regarded as "immunity gone w rong", requires no detailed description here. Furthermore, acquired hypersensitivity to molds and thermophyllic actinomycetes released in confined spaces may cause relatively acute manifestations, such as "farmer's lung" in man or conditions resembling "atypical" interstitial pneumonia, pulmonary emphysema and hypersensitivity pneumonitis in cattle and horses. Another hypersensitivity condition, known as facial excema of sheep, occurring in New Zealand and parts of Australia, results from photosensitization associated with hepatotoxicity after contact with a toxin of the fungus Pithomyces chartarum.

This fungus is said to proliferate most rapidly, on dead plant material and in ryegrass pastures, whenever hot humid weather follows a dry period. Traces of epithelial fragments from cats, horses, poultry etc. may also cause respiratory or generalized anaphylactic reactions in man and in animals; similar responses may be triggered by ingestion of or contact with nuts, beans, potatoes, cereal products, fish, shellfish, milk or egg-proteins and with other substances found commonly in the environment. Quite severe reactions have been precipitated by contamination of the environment by dusts introduced by air conditioning systems or heating ducts. Guidelines for the asthmatic patient during air pollution episodes were laid down by the Weather and Air Pollution Committee of the American Academy of Allergy in 1975.

\section{PROTOZOAN AND METAZOAN PARASITES}

In the time available, it is impossible to deal adequately with the complex interactions of environment, premunity and immunity in diseases caused by parasites. Only two examples will be given.

Widespread contamination of the environment with infective forms of Toxoplasma gondii, and its persistence for long periods in meat, results in infestation of numerous species of mammals and birds. The resultant disease is usually subclinical, but encephalitis, ataxia, myalgia, abortion and neonatal deaths may occur. In man, the disease often follows serious outbreaks of abortion in sheep. Its extensive distribution, and the fact that the organism is resistant to cold and relatively insensitive to heat, results in detectable antibody titres in more than $60 \%$ of sheep (Watson, W.A. 1973 Personnal Communication) and about $50 \%$ of some human populations. These (IgA and IgM) antibodies contribute to the elimination of organisms from the blood, but cell-mediated immunity is probably more effective in reducing infestation of tissues. Nevertheless, persistence of humoral antibody for long periods in man suggests continuing infection, and local inflammation evoked by a delayed hypersensitivity type reaction may play an important role in the eventual rejection of parasites of many types.

Immunity to nematodes is determined partly by the degree of contact between the host and the infective stages in the environment. This, in turn, is influenced to a great extent by climatic factors, by the ability of free-living stages to persist for long periods under the prevailing weather condition and by grazing intensity. When infestation occurs (e.g. with Ostertagia), subsequent loss of worms is proportional to the total number present, but a gradually increasing resistance to the establishment of new worms occurs. Thus, by the time that full resistance develops, about 20 times as many worms have been found in fully susceptible calves as are present in previously infected animals. Resistance to nematodes is manifested in numerous other ways, including structural modification of the parasites themselves, and reduction in their ability to develop fully, to lay eggs or to produce fully infective larvae. Environmental influences are also associated with the arrest, at certain stages of development, of the larval forms within a host (hypobiosis). This occurs especially in autumn and winter months, and a high proportion of "hypobiotic forms" subsequently fail to complete their life cycle in the spring. The biologic processes in both host and parasite which determine the onset and outcome of hypobiosis remain to be elucidated, but stimulation of the host's immune systems certainly occurs. 


\section{CONCLUSIONS}

In conclusion, then, it may be stated unequivocally that weather and the physical nature of the immediate environment, together with topographical factors, play an important part in determining the resistance of the population of a locality to disease. The genetic constitution of the individual interacts with these factors, in a positive or a negative manner, to enhance or minimize susceptibility, and further variability of basal immunity may be associated with the nutritional status in utero and during the neonatal period. The degree of environmental contamination with specific organisms (which is influenced greatly by climatic variations) may induce either a desirable degree of immunity to disease or, if the burden of pathogens is too great, may result in morbidity or mortality on an economically significant scale. Special considerations exist in the case of immature animás maintained indoors in systems of intensive husbandry. Under such conditions, poor ventilation, ineffective floor drainage, cold and damp may combine to reduce the efficacy of the mucociliary and other primary defence systems, whilst insufficient colostrum, overcrowding, rapid passage of organisms from animal to animal (especially when different age-groups are housed in proximity), and psychological and environmental stress all tend to reduce or overwhelm the developing humoral and cell-mediated defence mechanisms. Thereafter, throughout life heat, cold, humidity, altitude, dietary factors (frequently influenced by weather), and stresses of various kinds continue to alter an animal's ability to develop and maintain levels of humoral and cell-mediated immunity adequate to ward off infection. Further modification of the immune status of host populations is determined by the effects of weather on the availability of breeding grounds and on the life cycle of insect vectors of disease.

It is now evident that the relationships between weather, the environment and resistance to disease are extremely complex, and many interactions remain to be investigated fully. Since conditions of "high isolation" are seldom practicable for economic reasons, and perhaps not even desirable, the principal weapons remaining in the armoury of preventive medicine are epidemiological surveys, good hygiene, balanced diet, reduction of environmental contamination, mitigation of stresses, and immunologic pre-conditioning (possibly by multi-component or polyvalent vaccines). By these measures we may hope to reduce the losses caused by disease and thereby do much to meet the nutritional requirements of an expanding world population.

\section{RE FERE NCES}

a selected bibliography

ALLEN, T.R. (1973) : Common colds in Antarctica. J. Hyg., 71:649-651.

ALLEN, T.R., BRADBURNE, A.F., STOTT, E.J., GOODWIN, C.S. and TYRRELL, D.A.J.

(1973) : An outbreak of common colds in an Antarctic base after seventeen weeks of complete isolation. Ibid, 71:657-672.

BACKSTROM, L. (1973) : Environment and animal health in piglet production. Acta. vet. Scand. Suppl., $41: 1-240$.

BRUNSDON, R.V. (1973): In: Proceedings of the 19th Postgraduate Committee for Veterinarians, Sect. 19, University of Sydney, Sydney.

DOUGHERTY, J. (1971) : In: A Guide to Experimental Environmental Research on Animals. pp. 215-245. National Academy of Science, Washington, D. C.

EWER, T.K. (1974) : What do we mean by Stress? Vet. Rec., 95: 180-182.

FERNANDES, G., YUNIS, E.J., JOSE, D.G. and GOOD, R.A. (1973): Dietary influence on antinuclear antibodies and cell-mediated immunity in NZB mice. Int. Arch, Allergy Appl. Immunol., 44: 770-782.

GIBSON, T.E. (1975) : Helminthology. In Veterinary Annual Vol.15. Wright Scientechnica, Bristol .

GIBSON, T.E. and EVERITT, G. (1972) : The ecology of free living stages of Ostertagia circumcineta. Parasitology, $64: 451-460$.

HENNING, M.W. (1956) : In: Animal Diseases in South Africa, p. 735. Central News Agency, South Africa.

HYSLOP, N.St.G. (1969) : Developments in Veterinary Science: Bovine pulmonary emphysema. Canad. vet. J., 10:251-257.

HYSLOP, N.St.G. (1971) : Factors influencing the epidemiology and epizootiology of airborne diseases. J.A.V.M.A., 159: 1500-1507.

IPSEN, J. (1952)

: Effect of temperature on the immune response. J. Immunol., 69:273279 . 
JOSE, D. G. and GOOD, R.A. (1972): In: Proceedings of the 6th Leukocyte Culture Conference. Academic Press, New York.

JOSE, D.G., STUTMAN, O. and GOOD, R.A. (1973): Long-term effects on immune function of early nutritional deprivation. Nature, Lond., 241:57-58.

KAYSER, C. (1961) : In: The Physiology of Natural Hibernation. Pergamon Press, Oxford. KIRKPATRICK, C.H. (1975): Properties and activities of transfer factor. J. Allergy Clin, Immunol., $55: 411-421$.

LARSH, J.E. and WEATHERLY, N.F. (1974): Cell-mediated immunity in certain parasitic infections. In: Current Topics in Microbiology and Immunology, Vol.67; pp. 113-137. Springer-Verlag, Berlin.

MICHEL, J.F. (1973) : Osterlagia ostertagi - protective immunity in calves. Exptl. Parasit., $33: 179-186$.

NEWBERNE, P.M. (1973): In: Advances in Veterinary Science and Comparative Medicine, Vol.17, pp. 265-273. Academic Press, New York.

PARKER, D., SOMMER, G. and TURK, J.L. (1975): Variation in guinea pig responsiveness. Cell. Immunol., 18:233-238.

PAYNE, P.R. (1972) : In: Nutrition and Diseases in Experimental Animals, pp. 9-16. Bailliere, Tindall and Cox Ltd., London.

PE PYS, J. (1969) : In: Pulmonary Aspergillosis in Hypersensitivity Diseases of the Lungs due to Fungi and Organic Dusts. Karger A.G., Basel.

REYNECKE, J. and BONSMA, J.C. (1964): In: Proceedings of the South African Society for Animal Production, 1964 pp. 170-172. South African Society for Animal Production, Pretoria.

SCRIMSHAW, N.S., TAYLOR, C.F. and GORDON, J.E. (1968): Interactions of Nutrition and Infection. Wld. HIth. Org. Monograph Series 57. World Health Organization, Geneva.

SELL, S. (1972) : In: Immunology, Immunopathology and Immunity. Harper and Row Inc., Maryland.

SMITH, L. P., HUGH-JONES, M.E. and JACKSON, O.F. (1972): Weather conditions and disease. Vet. Rec., 91:642.

TROMP, S.W. (1973) : Meteorological changes and resistance to infection. Lancet (1) 1973, $1517-1518$.

TUMBELSON, M.E., BURKS, M. F. and WINGFIELD, W.E. (1973): Aging and serum proteins. Cornell Vet., $63: 65-71$.

WOODRUFF, J.F. (1970): The influence of quantitated post-weaning undernutrition on Coxsackievirus $\mathrm{B}_{3}$ infection of adult mice-II. Alteration of host defence mechanisms. J. infect. Dis., 121: 164-181. 